Т. Д. Вентцель, В. С. Владимиров, В. В. Жиков, А. М. Ильин, В. А. Ильин, В. А. Кондратьев, Л. Д. Кудрявцев, Е. Ф. Мищенко, С. М. Никольский, Ю. С. Осипов, Е. В. Радкевич, Н. Х. Розов, В. А. Садовничий, Л. Д. Фаддеев, Г. А. Чечкин, А. С. Шамаев, Т. А. Шапошникова, А. А. Шкаликов, Олейник Ольга Арсеньевна (некролог), УМH, 2003, том 58, выпуск 1, 165-174

DOI: https://doi.org/10.4213/rm607

Использование Общероссийского математического портала Math-Net.Ru подразумевает, что вы прочитали и согласны с пользовательским соглашением http://www.mathnet.ru/rus/agreement

Параметры загрузки:

IP: 34.229 .108 .108

26 апреля 2023 г., $14: 23: 16$ 


\section{ОЛЕЙНИК ОЛЬГА АРСЕНЬЕВНА}

13 октября 2002 года исполнился год со дня кончины Ольги Арсеньевны Олейник, действительного члена Российской академии наук, заведующей кафедрой дифференциальных уравнений механико-математического факультета МГУ им. М.В. Ломоносова. Российская наука понесла огромную утрату. Ушла из жизни замечательная женщина, выдающийся педагог и блестящий ученьй.

Ольга Арсеньевна Олейник родилась в городе Матусове Киевской области. Все ее детство прошло на Украине. Во время войны, в годы тяжелых испытаний, выпавших на долю нашей страны, Ольга Арсеньевна вынуждена была уехать в эвакуацию на Урал. Окончив среднюю школу в городе Перми в 1942, Олейник поступила на физико-математический

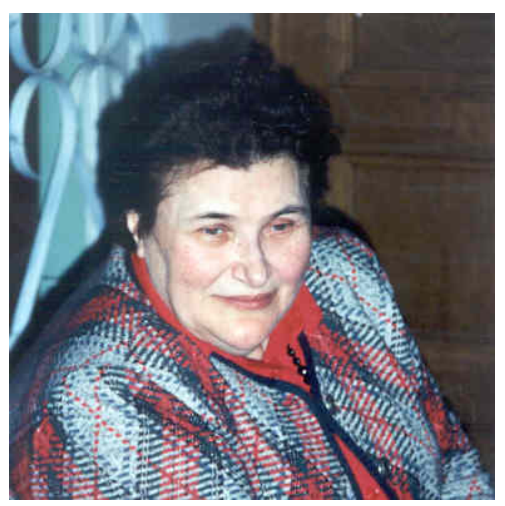
факультет Пермского университета. Способности Ольги Арсеньевны проявились очень рано и в 1944 году по рекомендации профессора МГУ С.А. Яновской она была переведена на механико-математический факультет Московского университета. С этого момента вся ее жизнь связана с МГУ.

После студенческих лет с 1950 года О.А. Олейник работает преподавателем на кафедре дифференциальных уравнений механико-математического факульета МГУ, сначала - ассистентом, затем доцентом, с 1955 года - профессором, а с 1973 года - заведующей кафедрой. С 1948 по 1962 гг. она работала по совместительству в Математическом институте имени В.А. Стеклова АН СССР, а с 1965 г. - в Институте проблем механики АН СССР (ныне Российской академии наук).

О. А. Олейник является всемирно известньм ученым. Ее перу принадлежит свыше 370 научных статей и 8 монограбфий. Тематика ее основных работ - топология алгебраических многообразий, уравнения с частными производными, математическая физика, теория пограничного слоя, теория упругости, теория усреднения.

Научная жизнь О. А. Олейник началась с первых студенческих лет. Она была активным участником семинара Ивана Георгиевича Петровского. Уже в эти годы О.А. Олейник получила несколько интересных и важных научных результатов. Так, она доказала [1], что в задаче Дирихле для произвольного линейного эллиптического уравнения второго порядка с гладкими коэффицциентами граничная точка регулярна тогда и только тогда, когда эта точка регулярна для уравнения Лапласа.

Окончив с отличием мех-мат в 1947 году, О.А. Олейник поступила в аспирантуру Института математики МГУ к И. Г. Петровскому. В 1950 году она защитила кандидатскую диссертацию на тему "О топологии действительных алгебраических кривых на алгебраической поверхности". В этой диссертации, а также в работах [2]-[9], часть которых выполнена совместно с И.Г. Петровским, было достигнуто существенное продвижение в XVI-й проблеме Гильберта. Получены оценки эйлеровой характеристики $(n-1)$-мерной алгебраической поверхности порядка $m$ 
в $n$-мерном проективном пространстве через число $\Pi_{n}(m)$ целых точек куба $[0, m-1]^{n}$, лежащих на центральном сечении куба гиперплоскостью, перпендикулярной главной диагонали. Величины $\Pi_{n}(m)$ получили в литературе название чисел Петровского-Олейник. А оценки, названные "неравенствами Петровского-Олейник", стали базой для многочисленных дальнейших исследований. В частности, из этих оценок вытекают результаты о возможном расположении связных компонент алгебраической поверхности в проективном пространстве. В работах [4], [6] получены оценки числа кусков алгебраической поверхности порядка $m$ в $n$-мерном проективном пространстве (многомерный аналог теоремы Харнака); позже подобные оценки были выведены также Р. Томом (Франция) и Дж. Милнором (США), причем в оценках последнего старшие члены, имеют тот же порядок, что и у Олейник, но большие коэффициенты.

В 1954 году Ольга Арсеньевна защитила докторскую диссертацию на тему "Краевые задачи для уравнений с частными производными с малым параметром при старших производных и задача Коши для нелинейных уравнений в целом" (автореферат опубликован в [22]).

Ольгой Арсеньевной получены результаты первостепенной важности в большинстве разделов современной теории уравнений с частными производными. При исследовании краевых задач для эллиптических уравнений второго порядка часто используется лемма о производной решения в точке граничного экстремума по некасательному к границе направлению. С. Зарембой в 1910 году эта лемма была доказана для гармонических функций: затем французский математик Ж. Жиро с помощью сложных построений распространил ее на случай эллиптических уравнений с переменными коэффициентами. В 1952 году выходит работа Ольги Арсеньевны [11], где дано элементарное доказательство этой леммы для решений общего линейного эллиптического уравнения второго порядка; другое простое доказательство одновременно и независимо появилось в статье Э. Хопфа (США). Эта лемма по праву носит имя леммы Олейник-Хопфа и входит во все основные курсы уравнений с частными производными.

Работы 1950-1952 годов О. А. Олейник [10], [12], [15] открывают новое научное направление в нашей стране, посвященное исследованию краевых задач для эллиптических и параболических уравнений второго порядка с малым параметром при старших производных.

Цикл работ 1954-1958 годов [18]-[20], [25]-[27], [30], [34], [36], [37] посвящен теории разрьвных решений нелинейных уравнений гиперболического типа. Такиезадачи возникают при исследовании моделей газовой динамики при наличии ударных волн. Для скалярного квазилинейного уравнения первого порядка, которое служит моделью системы уравнений газовой динамики, совместно со своим учеником С. Н. Кружковым Ольгой Арсеньевной была построена полная теория разрьвных решений: введено физически мотивированное понятие обобщенного решения задачи Коши; сформулирован принцип отбора допустимых разрьвных решений, аналогичньй условию возрастания энтропии; доказаны глобальное существование обобщенного решения задачи Коши, его единственность и непрерьвная зависимость от начальных данных; описаны структура множества точек разрьва обобщенного решения и его поведение при больших значениях времени; показано, что обобщенное решение может быть построено как предел решений параболических уравнений с мальм параметром при старшей производной (обоснован "методисчезающей вязкости", часто используемьй в прикладных задачах). В [28] была доказана единственность обобщенного решения задачи Коши для квазилинейной гиперболической системы, возникаюшей в ряде разделов механики. Работы О. А. Олейник вместе с исследованиями Э. Хопфа (США), П. Лакса (США), И. М. Гельфанда, А.Н. Тихонова, С. Н. Кружкова являются основой теории разрывных решений нелинейных гиперболических уравнений и систем.

Опубликованная в 1957 году статья [31] легла в основу математической теории нестационарной фильтрации, т.е. неустановившихся течений сжимаемых жидкостей и газов в пористых средах. Эти течения описьваются нелинейными уравнениями с так называемым неявным вырождением: например, возникают уравнения, имеющие второй порядок и параболический тип при положительных значениях искомой функции, а при ее обращении в нуль вырождающиеся в уравнения первого порядка. Для широкого класса нелинейных неявно вырождающихся параболических уравнений теории нестационарной фильтрации О. А. Олейник определение обобщенного решения задачи Коши, согласующееся с физическим смыслом рассматриваемых величин, доказала, что обобщенное решение существует и единственно, изучила его свойства. В дальнейших работах О.А. Олейник совместно со своим учеником А. С. Калашниковьгм сформулировала и доказала основополагающие результаты математической нестационарной фильтрации. В последу- 
ющие десятилетия эти результаты получили развитие в многочисленных работах отечественных и зарубежных ученых; в настоящее время данная тематика определяет одно из центральных направлений исследований в области нелинейных уравнений с частными производными.

В цикле работ О.А. Олейник 1963-1972 годов [53], [55]-[57], [69], [71]-[73], [75], [77]-[82], [86]-[88], [92], [97], [98] построена математическая теория пограничного слоя. Уравнения пограничного слоя были предложены 4 известным немецким механиком Л. Прандтлем еще в 1904 году, затем на протяжении почти 60 лет рассматривались многими авторами, однако основные принципиальные вопросы математического характера, связанные с этими уравнениями, оставались открытьми. Ольга Арсеньевна впервые доказала существование решений основных краевых задач для стационарных и нестационарных систем уравнений, описывающих пограничньй слой в несжимаемой вязкой жидкости, получила теоремы единственности, исследовала устойчивость решений относительно возмущений внешнего течения, начальных данных и поверхности обтекаемого тела, изучила асимптотические свойства решений, обосновала методы их приближенного построения. Эти и другие результаты вошли в монографию [89]. На основе совместных работ О.А. Олейник и ее ученика В. Н. Самохина написана книга [357], см. также [368].

В 1964-1971 годах выходит серия работ Ольги Арсеньевны [60], [62], [70], [74], [94]-[96], где построена теория линейных уравнений второго порядка с неотрицательной характеристической формой, т.е. эллиптических уравнений, которые могут произвольным образом вырождаться на любых подмножествах области, где ищется решение, и ее границы. Для общего уравнения с неотрицательной характеристической формой О.А. Олейник доказала единственность обобщенного решения первой краевой задачи; этим был дан, ответ на вопрос, поставленньй известным итальянским математиком Г. Фикерой. Ольга Арсеньевна доказала также существование обобщенного решения этой задачи методом эллиптической регуляризации, исследовала гладкость обобщенного решения, получила совместно со своим учеником Е. В. Радкевичем для общих уравнений с неотрицательной характеристической формой достаточные условия гипоэллиптичности, установила их необходимость в случае аналитических коэффициентов. Эти и другие результаты изложены в монографии [93], переведенной на английский, итальянский и, китайский язьщи.

В 1972-1975 годах О.А. Олейник и Е. В. Радкевич получили [99]-[101], [103]-[105], [109], [116]-[121] ряд глубоких результатов об аналитичности решений линейных уравнений и систем. Эта тематика восходит к XIX-й проблеме Гильберта. О.А. Олейник и Е.В. Радкевич применили новьй подход, установив априорную оценку аналитического продолжения решения в комплексную область для уравнений и систем, допускающих только аналитические решения. С помощью этой оценки были выделены широкие классы систем, имеющих неаналитические решения, а также найдены новые классы систем, все решения которых аналитичны. Также в эти годы Олейник совместно с Радкевичем изучает свойства решений различньх уравнений и систем в неограниченных областях. В работах [107], [108], [110], [113], [118]-[120] изучаются общие линейные эллиптические и параболические системы в неограниченных областях. При этом вводится дополнительная независимая переменная, относительно которой все решения аналитичны. Этим методом были найдены классы единственности решений обших краевых задач в неограниченных областях, доказаны теоремы типа Фрагмена-Линделефа и Лиувилля, получены оценки собственных функций общих эллиптических краевых задач, установлена единственность решений краевых задач без начальных условий для параболических систем. По-видимому, возможности этого метода еще далеко не исчерпаны. Еще один подход, применявшийся О.А. Олейник к изучению стационарных и эволюционных уравнений в неограниченных областях, связан с доказанными ею совместно с ее учеником Г. А. Иосифьяном обобщениями так называемого принципа Сен-Венана, о котором подробнее будет сказано ниже в связи с работами Ольги Арсеньевны по теории упругости. На этом пути исследовано поведение решений линейньх эллиптических уравнений в окрестности бесконечности, а также получены новые теоремы о единственности обобщенных решений первой краевой задачи для линейных параболических и эллиптических уравнений второго порядка в классах растущих функций, причем допустимая скорость их роста тем больше, чем быстрее рассматриваемая область сужается на бесконечности [136], [143], [171], [172].

В 1975-1978 годах О.А.Олейник предложила новый метод исследования эволюционных уравнений [125], [126], [159], [160]. Метод основан на введении параметра в рассматриваемое уравнение (или систему) и априорных оценках, его решений в ограниченной области. Этим методом бы- 
ли доказаны теоремы о единственности решений общих краевых задач в неограниченных областях для параболических систем, обобщающие известные результаты Э. Хольмгрена, А. Н. Тихонова и С. Тэклинда о задаче Коши для уравнения теплопроводности; найдены оценки, характеризующие поведение функций Грина общих краевых задач на бесконечности и вблизи начальной гиперплоскости; исследованы некоторые классы уравнений, не разрешенных относительно старшей производной по времени.

Очень интересны работы О. А. Олейник о поведении решений на бесконечности. Эта тематика занимала Ольгу Арсеньевну на протяжении многих лет и оставалась одной из основных практически до конца жизни.

Для некоторых классов полулинейных эллиптических уравнений второго порядка Ольгой Арсеньевной совместно с В. А. Кондратьевым исследовано [305], [306], [308], [345], [357] поведение на бесконечности решений, заданных в неограниченном или полуограниченном цилиндре и удовлетворяющих на его боковой поверхности однородному условию Дирихле или Неймана. В частности, описаны все возможные виды асимптотики при $x_{n} \rightarrow \infty$ заданных в цилиндре решений уравнения $\Delta u+k u_{X_{n}}-|u|^{p-1} u=0(p>1, k \in \mathbb{R})$, представляющего большой интерес для теории пограничного слоя. Эти же вопросы исследованы в случае уравнения $\Delta u-e^{u}=0$, модельного для многих задач математической физики и геометрии. В совместной работе О. А. Олейник и испанского математика Х. И. Диаса [307] для определенного класса полулинейных эллиптических уравнений доказаны существование и единственность обобщенного решения смешанной краевой задачи (в частности, задачи Дирихле или Неймана) в неограниченной области без каких-либо предположений о росте решения и свободного члена уравнения на бесконечности. Асимптотические свойства решений эллиптических и параболических систем в цилиндрических областях исследованы в [365]. Многие результаты О.А. Олейник, относящиеся к нелинейным уравнениям в неограниченных областях, изложены в ее монографии [322].

Работы О.А. Олейник по математической теории упругости начинают выходить с 1976 года. Они публикуются частично в соавторстве с В. А. Кондратьевьм, а также со своими учениками Г. А. Иосифояном, И. Копачеком и др. В этом разделе механики важную роль играет открытьй более ста лет назад принцип Сен-Венана, согласно которому энергия, сосредоточенная в части самоуравновешенного упругого тела, далекой от зоны приложения внешних сил, должна быть малой. Как известно, ряд задач плоской теории упругости связан с изучением бигармонического уравнения. В работе [154] дано новое доказательство принципа Сен-Венана для бигармонического уравнения, учитьвающее геометрию области; лежащий в основе этого доказательства метод весовых функций нашел в дальнейшем многочисленные применения. Как следствие в [154] получены теоремы единственности решений основной краевой задачи плоской теории упругости в неограниченных областях, а также описано поведение решений на бесконечности, в окрестности нерегулярных точек границы и в окрестности особых точек, лежащих внутри области. В работах [177], [178], [187] доказан принцип Сен-Венана для системы уравнений теории упругости при наиболее общих предположениях (наличие объемных сил, нецилиндрическая область); на его основе решен давно поставленный вопрос об условиях, гарантирующих экспоненциальное убьвание на бесконечности решений системы теории упругости с конечной энергией в неограниченном цилиндре. В двумерной теории упругости возникает необходимость изучения краевых задач для бигармонического уравнения в областях с нерегулярньми границами. Работы [204], [207], [211], [228], [293] содержат неулучшаемые оценки в окрестности нерегулярных граничных точек решений задачи Дирихле для бигармонического уравнения в плоских областях с негладкими границами, причем допускаются ненулевые граничные условия и сингулярные обобщенные функции в качестве свободных членов. В частности, окончательно решена давно стоявшая проблема о характере особенности, которую может иметь решение бигармонического уравнения в концевой точке трещины. Наряду с оценками решений выведены [213], [257], [258] оценки их первых и вторых производных.

Одну из важных ролей при изучении краевых задач для системы уравнений теории упругости играют неравенства Корна, связьвающие интеграл Дирихле, энергию и $L_{2}$-норму решения. В случае ограниченной области такие неравенства доказьвали различными методами многие авторы, начиная с А. Корна, работы которого относятся к 1906-1909 гг. Совместно с В. А. Кондратьевым Ольга Арсеньевна предложила элементарные доказательства неравенств Корна и их обобщений, пригодные как для ограниченных, так и для некоторых классов неограниченных об- 
ластей. При этом была явно вьписана зависимость постоянных, содержащихся в этих неравенствах, от формы и размеров области [266], [277], [284], [295], [303], [304]. С помощью доказанных неравенств О.А. Олейник и В. А. Кондратьев исследовали различные краевые задачи для системы теории упругости в неограниченных областях. Для задачи Дирихле в [277] развит также другой подход к исследованию единственности решения, основанный на введенном авторами обобщении понятия емкости. Кроме того, в [277] доказано существование решения задачи Дирихле для системы теории упругости в неограниченной области. Дальнейшее развитие этих результатов содержится в [286], [289], [292]. В работах [299], [302] с помощью неравенств типа Корна для системы теории упругости в неограниченных областях исследованы классические краевые задачи Буссинеска и Черрути, в которых для разных компонент решения ставятся разные типы граничных условий. Отметим еще работы [321], [326], [318], где получены неравенства Корна для областей типа решеток и соединений, которые зависят от нескольких малых параметров и могут иметь сложную геометрическую структуру, причем постоянные в вьведенных неравенствах являются асимптотически точньми по отношению к этим параметрам.

С конца 70-х годов О.А. Олейник начинает заниматься задачами теории усреднения диффференциальных операторов. Ее можно по праву считать одним из создателей этой области математики. Теория усреднения возникла на рубеже $60-\mathrm{x}$ и $70-\mathrm{x}$ и начала интенсивно развиваться в связи с прикладньми задачами механики сильнонеоднородных сред, проблемами материаловедения (композиционные и армированные материалы, пористые материалы), почвоведения, нефбтеразработки, производства космических кораблей и антенн и многих других. Такие задачи приводят к дифференциальным уравнениям, неудобным для непосредственного исследования ввиду быстрой осцилляции коэффициентов или большой сложности границы области. Поэтому возникает проблема построения усредненного уравнения, имеющего постоянные или медленно изменяющиеся коэффициенты и обладающего решениями, близкими в той или иной норме к соответствуюшим решениям исходного уравнения; для этого требуется произвести асимптотический анализ задачи. С теорией усреднения тесно связан вопрос о $G$-сходимости семейства зависящих от малого параметра операторов, которая означает слабую сходимость обратных операторов. Ольгой Арсеньевной (совместно с В.В.Жиковым и С. М. Козловым) построена [164], [169], [176] теория $G$-сходимости эллиптических и параболических уравнений произвольного порядка; изучены свойства $G$-сходящихся операторов; введено понятие сильной $G$-сходимости; найдено необходимое и достаточное условие $G$-сходимости дифференциальных операторов, позволяющее в ряде случаев явно выписать $G$-предел; даны оценки коэффициентов оператора, являющегося $G$-пределом семейства дифференциальных операторов; доказана сходимость соответствующих интегралов энергии; исследована $G$-сходимость дифференциальных операторов со случайными и почти периодическими коэффициентами. В [182], [186] для параболических уравнений с почти периодическими коэффициентами предложен другой подход к проблеме усреднения, основанный на понятии почти решения вспомогательного уравнения. Проблема усреднения для системы теории упругости с почти периодическими коэффициентами решена в [196]. В связи с некоторыми задачами теории усреднения французским математиком Э. Санчес-Паленсией был поставлен вопрос о поведении на бесконечности решений эллиптического уравнения второго порядка, периодических по части независимых переменных и обладающих конечным интегралом Дирихле по периоду. Этот вопрос решен в работах [221], [226], [239], вьполненных Ольгой Арсеньевной совместно с В.А. Кондратьевым. Они исследовали также аналогичные вопросы для параболического уравнения второго порядка [229] и для эллиптических систем [230], [261] Ольгой Арсеньевной рассмотрен [273]-[276], [278], [290], [297], [300] ряд спектральных задач, возникающих при описании собственных колебаний сильнонеоднородных упругих тел.

Работа [275] посвящена исследованию задачи о собственных колебаниях струны с концентрированной массой, там Ольга Арсеньевна совместно со своими учениками Ю. Д. Головатьм и Т. С. Соболевой, а также С.А. Назаровым получила полные асимптотические разложения собственных значений и собственных функций относительно малого параметра, которым является длина отрезка, где сосредоточена концентрированная масса. В [290] эти результаты распространены на многомерньй случай.

В статье [285], написанной О.А. Олейник совместно с ее учениками Г.А. Иосифьяном и А. С. Шамаевым, предложен новьй подход к спектральным задачам теории усреднения, базирующийся на доказанных авторами общих теоремах о поведении спектра семейства, абстрактных 
операторов, которые зависят от параметра и действуют в различных пространствах, также зависящих от параметра. Этот подход имеет широкие применения. Многие из них изложены, в третьей главе книги [291] (см. также [319]): дан асимптотический анализ собственных значений и собственных функций краевых задач теории упругости для сильнонеоднородных сред; исследовано, поведение собственных значений и собственных функций задачи Дирихле в перфорированной области для эллиптического уравнения, что приводит к задаче усреднения для эллиптического уравнения, вырождающегося на границах полостей; изучены спектральные задачи для эллиптических уравнений в областях с быстроосциллирующими границами; описано поведение собственных значений и собственных функций краевых задач для уравнения Пуассона в области с отверстиями малой суммарной концентрации; получены новые доказательства ряда теорем о собственных колебаниях упругих тел с концентрированными массами.

Совместно со своим учеником Г. А. Чечкиным О.А. Олейник рассмотрела [323], [339], [341], [342] для неоднородного эллиптического уравнения второго порядка краевую задачу в области из $\mathbb{R}^{n}$ с быстро меняющимся типом граничных условий, т.е. нулевым краевым условием Дирихле на одной части границы и нулевьм краевьм условием третьего рода (условием Фурье) на остальной ее части в предположении, что последняя состоит из многих изолированных компонент, причем число их неограниченно возрастает, а диаметры стремятся к нулю; здесь малым параметром $\varepsilon$ является наибольший из этих диаметров. В [323] установлено, что если число компонент второй части границы, возрастает при $\varepsilon \rightarrow 0$ не слишком быстро, т.е. как $|\ln \varepsilon|^{\gamma}, 0<\gamma<n-1$, то обобщенные решения смешанной задачи сходятся к обобщенному решению задачи Дирихле с нулевым условием на всей границе; выведены оценки для разности между допредельной и предельной функциями в различных нормах. Изучена также соответствующая спектральная задача. Аналогичное исследование проведено для системы теории упругости [339], [341], [342], при этом пришлось доказывать неравенство типа Корна для нового класса областей.

В статьях [314], [316], написанных совместно с А. С. Шамаевьм и немецким математиком В. Егером, изучена асимптотика решения уравнения Пуассона в частично перфорированной области с нулевым краевым условием Неймана на границах полостей и нулевым условием Дирихле на внешней границе; изучена также соответствующая спектральная задача. В [328], [343], [359] рассмотрены эти же вопросы для случая, когда на границах полостей ставится нулевое краевое условие третьего рода, зависящее от параметра. В совместных работах О.А. Олейник, Г. А. Иосифьяна и французского математика Р. Темама [329], [333] исследована задача Дирихле для эллиптических уравнений второго порядка, которые содержат быстроосциллпрующие члены, нелинейные относительно искомой функции и ее градиента.

В цикле работ О. А. Олейник и Т. А. Шапошниковой [330], [331], [334], [336], [361]-[364] исследованы краевые задачи для уравнения Лапласа в частично перфорированных областях. В случае задачи Дирихле рассмотрены частично перфорированные области общего вида с непериодической структурой; в случае смешанной задачи с зависящим от параметра краевым условием получены оценки отклонения решения от решения предельной задачи, вид которой зависит от порядков малости параметра, входящего в граничное условие, и параметра, характеризующего периодическую структуру. Изучены также частично перфорированные области с полостями малой плотности и полостями, размещенными вдоль гиперповерхности. В статьях [348], [349], [369] рассматриваются вопросы усреднения операторов в области, часть которой содержит периодически расположенные тонкие каналы. Вопросы усреднения дифференциальных уравнений высокого порядка (бигармоническое уравнение) в перфорированной области рассмотрены в [346], [347].

Многие результаты О.А. Олейник по теории усреднения изложены в монографиях [315], [313] (соавторы В. В. Жиков и С. М. Козлов), [291], [319] (соавторы Г. А. Иосифоянн и А. С. Шамаев), а также [322]. Последняя написана на основе цикла лекций, прочитанных О. А. Олейник в 1993 г. в Риме по приглашению Специального комитета Итальянской национальной Академии деи Линчеи и ее президента.

В 1996-1997 годах выходят две работы [352], [355], посвященные исследованию задач типа Стефана (задач со свободной границей), которые возникают в теории вероятностей. Доказьвается теорема существования решений, а также теорема единственности.

Научные достижения О. А. Олейник были оценены по достоинству. Она была избрана действительным членом Российской академии наук, иностранным членом Итальянской национальной Академии деи Линчеи, иностранным членом Академии в Палермо, иностранным членом Акаде- 
мии наук и искусств в Милане (институт Ломбарде), иностранньм членом Саксонской академии наук, почетным членом Эдинбургского королевского общества, членом Европейской академии наук, была одним из учредителей Международного общества взаимодействия механики и математики. Ольге Арсеньевне присвоено звание почетного доктора Римского университета (doctor honoris causa).

В 1952 г. О.А. Олейник была присуждена премия имени Н. Г. Чеботарева за исследования по эллиптическим уравнениям с малым параметром при старших производных, в 1964 г. она была награждена премией имени М.В. Ломоносова первой степени за работы по теории пограничного слоя, в 1995 г. - премией имени И.Г. Петровского за исследования асимптотических свойств решений задач математической физики, а в 1988 г. удостоена Государственной премии за цикл работ "Исследования краевых задач для дифференциальных операторов и их приложения в математической физике", опубликованных в 1959-1985 гг. За заслуги в научно-педагогической деятельности О.А. Олейник в 1985 г. присвоено почетное звание заслуженного деятеля науки Российской Федерации.

Заслуги перед наукой и государством были высоко оценены. В 1995 г. издан указ Президента Российской Федерации о награждении О.А. Олейник орденом Почета. Также Ольга Арсеньевна награждена именной медалью Коллеж де Франс, медалью первой степени Карлова университета в Праге, Международной золотой медалью "Дайдалон в области математических наук" Междисциплинарного олимпийского форума в Афинах.

Многократно Ольга Арсеньевна приглашалась в качестве пленарного докладчика на конгрессы и международные конференции, а также для чтения лекций в ведущие университеты и научные учреждения различных стран Европы, Азии, Америки.

Широкую известность получил научно-исследовательский семинар по уравнениям с частными производными и математической физике, работавший на протяжении многих лет под руководством Ольги Арсеньевны в Московском университете. Докладчиками на этом семинаре были многие видные отечественные и зарубежные ученые.

О.А. Олейник подготовила 57 кандидатов физико-математических наук. Из ее учеников 20 стали докторами наук и профессорами, членами академий наук России и других стран. Под руководством Ольги Арсеньевны выполнили и защитили диссертации: М.В. Балабанов (Грузия), А. Г. Беляев, Н.М. Бокало (Украина), Н. И. Бриш (Белоруссия; впоследствии доктор наук, профессор; руководство совместно с И.Г. Петровским), Н.Д. Введенская, Т. Д. Вентцель, А. Г. Гагнидзе (Грузия), Т. Генчев (Болгария; впоследствии доктор наук, профессор), Ю. Д. Головатьй (Украина), Гу Лянькунь (Китай; впоследствии доктор наук, профессор), Т. Д. Джураев (Узбекистан; впоследствии доктор наук, профессор, действительньй член Академии наук Узбекистана), Ю. В. Егоров (впоследствии доктор наук, профессор), Е. П. ЖКидков (впоследствии доктор наук, профессор; руководство совместно с И. Г. Петровским), А. М. Ильин (впоследствии доктор наук, профессор, действительный член РАН), Г. А. Иосифьян, Р. Р. Кадыров (Узбекистан), А. С. Калашников (впоследствии доктор наук, профессор), С. Л. Каменомостская (С. Камин, Израиль; впоследствии доктор наук, профессор), Я.И. Канель, К. И. Карапетян (Армения; руководство совместно с И. Г. Петровским), И. Копачек (Чехия), С. Н. Кружков (впоследствии доктор наук, профессор), Л.П. Купцов, С.П. Левашкин, Д.М. Леквеишвили (Грузия), Н. О. Максимова; М. Маринов (Болгария), Т. А. Мельник (Украина; впоследствии доктор наук), В.П. Михайлов (впоследствии доктор наук, профессор; руководство совместно с И.Г. Петровским), Т. В. Михайлова, Г.К. Намазов (Азербайджан; впоследствии профессор), А. И. Насруллаев, А.В. Неклюдов, Б. Б. Оразов, В. Петков (Болгария; впоследствии доктор наук, профессор), Л. Я. Пьянзина, Е. В. Радкевич (впоследствии доктор наук, профессор), Н. У. Рахманов (Узбекистан), Е. С. Сабинина (Соболева), В. Н. Самохин (впоследствии доктор наук), Д. А. Силаев, Г. Н. Смирнова, Т. С. Соболева (впоследствии профессор), Е. Г. Соколова, В. И. Сукретньй (Украина), А. И. Суслов, И. Н. Тавхелидзе (Грузия), А. К. Тюлина, Г. М. Фатеева, Ха Тьен Нгоан (Вьетнам), Т. Уличевич (Югославия), Г. И. Хилькевич (Латвия), Г. А. Чечкин, Чжоу Юйлинь (Китай; впоследствии доктор наук, профессор, действительньй член Китайской Академии наук), С. Ф. Чичоян (Армения), А. С. Шамаев (впоследствии доктор наук, профессор), Т. А. Шапошникова (впоследствии доктор наук, профессор).

На протяжении многих лет Ольга Арсеньевна читала в Московском университете общий курс уравнений с частными производными, а также ряд специальных курсов. Написанный ею учеб- 
ник [137] широко используется преподавателями и студентами. В настоящее время готовится переиздание этого учебника в расширенном и переработанном виде в серии "Классический университетский учебник" к юбилею университета. По предложению О.А. Олейник в 1973 г. был организован семинар имени И.Г. Петровского по дифференциальным уравнениям и математическим проблемам физики. Начиная с 1978 г. по инициативе Ольги Арсеньевны проводятся ежегодные совместные сессии семинара имени И.Г. Петровского и Московского математического общества. В программу каждой из этих сессий включается, более ста докладов московских и иногородних ученых об их новых научных результатах по теории дифференциальных уравнений, математической физике, алгебраической геометрии, функциональному анализу, а также по другим разделам современной математики и ее приложений. Особенно представительной была сессия 2001 г., имевшая статус международной конференции, посвященной 100-летию со дня рождения И.Г. Петровского. На этой конференции было представлено более 500 докладов, резюме которых были опубликованы в специальном томе, вышедшем к началу конференции.

Ольга Арсеньевна была редактором переводов ряда монографий и учебников крупных зарубежных ученых: Р. Куранта, К. Миранды, Ж.-Л. Лионса, С. Мизохаты, Л. Берса, Ф. Джона, М. Шехтера, Э. Санчес-Паленсии, Ф. Сьярле. В 1986-1987 гг. опубликованы два тома "Избранных трудов" И. Г. Петровского, подготовленные к изданию Ольгой Арсеньевной. По инициативе Ольги Арсеньевны в них наряду с основными работами И. Г. Петровского включены развернутые комментарии к этим работам, содержащие обзоры более поздних исследований в соответствующих областях; несколько таких комментариев составлено при непосредственном участии О.А. Олейник [240], [241], [249]-[251]. В 1988 г. под редакцией Ольги Арсеньевны переиздана классическая монография С. Л. Соболева "Некоторые применения функционального анализа в математической физике". О.А. Олейник являлась главньм редактором сборников "Труды Московского математического общества" и "Труды семинара имени И.Г. Петровского", членом редакционных коллегий журналов "Дифференциальные уравнения", "Вестник Московского университета. Серия 1. Математика, механика", "Applicable Analysis" (США), "Rendiconti Lincei: Matematica e Applicazioni" (Италия), "Proceedings of the Royal Society of Edinburgh" (Великобритания). В течение многих лет она была заместителем главного редактора (тем, кто в немецком язьке называется Geschäftsführende Director) журнала "Успехи математических наук".

Ольгу Арсеньевну всегда отличала сила духа, высокая работоспособность, талант организатора, ответственное отношение ко всякому делу. При этом она оставалась женщиной, отзьвчивьм человеком, приятньм собеседником, другом.

Т. Д. Вентцель, В. С. Владимиров, В.В. Жиков, А. М. Ильин, В.А. Ильин, В. А. Кондратьев, Л. Д. Кудрявцев, Е. Ф. Мищенко, С. М. Никольский, Ю. С. Осипов, Е. В. Радкевич, Н. Х. Розов, В. А. Садовничий, Л. Д. Фаддеев, Г. А. Чечкин, А. С. Шамаев, Т. А. Шапошникова, А. А. Шкаликов

\section{СПИСОК НАУЧНЫХ ТРУДОВ О. А. ОЛЕЙНИК}

[339] On asymptotics of solutions and eigenvalues of the boundary value problems with rapidly alternating boundary conditions for the system of elasticity // Atti Accad. Naz. Lincei. 1996. V. 7. № 1. P. 5-15 (with G. A. Chechkin)

[340] On the homogenization of the Poisson equation in partially perforated domains with arbitrary density of cavities and mixed conditions on their boundaries // Atti Accad. Naz. Lincei. 1996. V. 7. № 3. P. 129-146 (with T. A. Shaposhnikova)

[341] О задачах усреднения // Университеты России. Фундаментальные проблемы математики и механики. Т. 1. М.: Изд-во МГУ, 1994. С. 223-226 (совм. с Г. А. Чечкиным, А. С. Шамаевым)

Начало списка см. в кн.: Труды семинара имени И.Г. Петровского. М.: Изд-во Моск. ун-та, 1987. Вьп. 12. С.9-19 и Труды семинара имени И. Г. Петровского. М.: Изд-во Моск. ун-та, 1996. Вып. 19. С. 20-25. 
[342] О поведении решений и собственных значений краевых задач с быстро меняющимися граничными условиями для системы теории упругости // Современные проблемы механики сплошной среды. Ростов-на-Дону: МП "Книга", 1995. С. 167-172 (совм. с Г. А. Чечкиным)

[343] On a homogenization problem for the Laplace operator in a partially perforated domain with the Neumann condition on holes // Proceedings of the Second Workshop on Composite Media and Homogenization Theory. Trieste: World Scientific, 1995. P. 167-188 (with W. Jäger, A. S. Shamaev)

[344] On some homogenization problems in partially perforated domains // Trends in Applications of Mathematics to Mechanics, Lisbon, 1994. London: Harlow, 1995. P. 220-277. (Pitman Monogr. Surveys Pure Appl. Math. V. 77.)

[345] On asymptotics of solutions of nonlinear second order elliptic equations in cylindrical domains // Partial Differential Equations and Functional Analysis in Memory of Pierre Crisvard. Boston: Birkhäuser, 1996. P. 160-173 (with V. A. Kondratiev)

[346] Об усреднении бигармонического уравнения в области, перфорированной вдоль многообразий малой размерности // Диффференц. уравнения. 1996. Т. 32. №6. С. 830-842 (совм. с Т. А. Шапошниковой)

[347] О задаче Дирихле для бигармонического уравнения в области, перфорированной вдоль многообразия малой размерности // Докл. РАН. 1996. Т. 350. №6. С. 742-746 (совм. с Т. А. Шапошниковой)

[348] Об усреднении оператора Лапласа в области, часть которой содержит периодически расположенные каналы с условиями Неймана на их границе // Вестник МГУ. Сер. матем., мех. 1996. № 5 (совм. с Ж. Тронелем, Т. А. Шапошниковой)

[349] Об усреднении смешанной задачи для уравнения теплопроводности в области, часть которой содержит тонкие, периодически расположенные каналы // Труды семинара им. И.Г. Петровского. 1996. Т. 20. С. 27-47 (совм. с Т. А. Шапошниковой)

[350] Роль теории дифференциальных уравнений в современной математике и ее приложениях // Соросовский образовательньй журнал. 1996. № 4. С. 114-121

[351] Об асимптотике при больших значениях времени решений нелинейных эволюционных уравнений и систем // УМН. 1996. Т. 51. № 5. С. 159-160 (совм. с В. А. Кондратьевым)

[352] О задачах со свободной границей, возникающих в теории вероятностей (теоремы единственности) // УМН. 1996. Т. 51. № 6. С. 205-206 (совм. с Г. Каллианпуром)

[353] Об усреднении решений уравнения Пуассона в областях, перфорированных вдоль гиперплоскости // Труды ММО. 1996. Т. 57. С. 47-71 (совм. с В. Егером, Т. А. Шапошниковой)

[354] On homogenization of solutions of the Poisson equation in a perforated domain with different types of boundary conditions on different cavities // Appl. Anal. 1997. V. 65. № 3-4. P. 205-223 (with W. Jäger, T. A. Shaposhnikova)

[355] О задачах со свободной границей, возникающих в теории вероятностей (теоремы существования) // УМН. 1997. Т. 52. № 1. С. 229-230 (совм. с Г. Каллианпуром)

[356] On spectral problems in partially perforated domains // Homogenization and Applications to Material Sciences. Tokyo: Gakkōtosho, 1997. P. 323-339

[357] Математические методы в теории пограничного слоя. М.: Наука, 1997 (совм. с В. Н. Самохиньпм)

[358] Анатолий Платонович Прудников (в честь семидесятилетия) // Дифференц. уравнения. 1997. Т. 33. № 6. С. 730-736 (совм. с С. В. Емельяновым, Ю. И. Журавлевым, В. А. Ильиным, Е. И. Моисеевым, Ю. В. Прохоровым, А. А. Самарским)

[359] Об асимптотике решений краевой задачи для уравнения Лапласа в частично перфорированной области с краевыми условиями третьего рода на границах полостей / / Труды ММО. 1997. Т. 58. С. 187-223 (совм. с В. Егером, А. С. Шамаевым)

[360] Юрий Алексеевич Митрополский (к восьмидесятилетию со дня рождения) // УМН. 1997. Т. 52. № 1. С. 237-239 (совм. с В. С. Владимировым, Е. Ф. Мищенко, Ю. С. Осиповым, А. М. Самойленко, К. В. Фроловым)

[361] О граничных задачах в областях, перфорированных вдоль многообразий // УМН. 1997. Т. 52. № 4. С. 205-206 (совм. с М. Лобо, М. Е. Перес, Т. А. Шапошниковой) 
[362] On homogenization of solutions of boundary value problems in domains / Perforated Along Manifolds. Dedicated to Ennio De Giorgi. Ann. Scuola Norm. Sup. Pisa Cl. Sci. (4). 1998. V. 25. № 3-4. P. 611-629 (with M. Lobo, M. E. Perez, T. A. Shaposhnikova)

[363] Об усреднении решений уравнения Пуассона в области, перфорированной вдоль гиперповерхности со смешанным краевым условием на границе полостей // Труды ММО. 1998. Т. 59. С. 150-174 (совм. с В. Егером, Т. А. Шапошниковой)

[364] Об усреднении краевых задач в перфорированных областях с непериодической структурой // Дифференц. уравнения. 1998. Т. 34. № 5. С. 647-661 (совм. с Т. А. Шапошниковой)

[365] Асимптотические свойства решений эллиптических и параболических систем в цилиндрических областях // Матем. сб. 1998. Т. 189. №3. С. 45-68 (совм. с Ю.В. Егоровым, В.А. Кондратьевым)

[366] Об усреднении краевых задач для эллиптических операторов в перфорированных областях с непериодической структурой // УМН. 1998. Т. 52. № 6. С. 179-180 (совм. с Т. А. Шапошниковой)

[367] Юрий Владимирович Егоров (к шестидесятилетию со дня рождения) // УМН. 1999. Т. 54. № 2. С. 195-204 (совм. с М. И. Вишиком, Л. Р. Волевичем, А. М. Ильиным, А. С. Калашниковым, В. А. Кондратьевым)

[368] Mathematical Models in Boundary Layer Theory. Boca Raton, FL: Chapman \& Hall/CRC, 1999 (with V. N. Samokhin)

[369] On homogenization of a boundary value problem in a domain with thin channels of a small length // Homage to Gaetano Fichera. Rome: Aracne, 2000. P. 289-304. (Quad. Mat. V. 7.) (with T.A. Shaposhnikova)

[370] Об усреднении краевых задач в областях с быстро осциллирующей непериодической границей // Дифференц. уравнения. 2000. Т. 36. № 6. С. 754-764 (совм. с В. Егером, Т. А. Шапошниковой)

[371] Линейные уравнения второго порядка параболического типа // Труды семинара им. И.Г. Петровского. 2001. Т. 21. С. 9-193 (совм. с А. М. Ильиным, А. С. Калашниковым) 